\title{
The Role of MicroRNA-381 in Chondrogenesis and Interleukin-1- $\beta$ Induced Chondrocyte Responses
}

\author{
Changhe Hou Fangang Meng Zhiqi Zhang Yan Kang Weishen Chen \\ Guangxin Huang Ming Fu Puyi Sheng Ziji Zhang Weiming Liao \\ Department of Joint Surgery, First Affiliated Hospital of Sun Yat-sen University, Guangzhou, \\ Guangdong, China
}

\section{Key Words}

Osteoarthritis • Chondrogenesis $\cdot$ Cartilage degeneration $・$ MiR-381 Metalloproteinase-13

\begin{abstract}
Aim: The molecular pathways regulating cartilage degradation are unclear. miR-381 was identified as a putative regulator of chondrogenesis related genes. Here, we examined its role in chondrogenesis and osteoarthritic cartilage degeneration. Methods: miR-381 expression was assessed in vitro in response to IL-1 $\beta$ stimulation in primary human $(\mathrm{PHC})$ and mouse (PMC) chondrocytes, and ATDC5 derived chondrocytes; and in vivo in mouse embryos and human osteoarthritic cartilage. The effects of miR-381 on chondrogenesis and NF-kB signaling were assessed using a synthetic RNA mimic or inhibitor and luciferase assay, respectively. Upstream regulators of miR381 were probed using siRNA or overexpression plasmids for Sox9 and Runx2. Results: miR-381 expression was elevated in chondrogenic and hypertrophic ATDC5 cells. miR-381 was induced in vitro by IL-1 $\beta$ in ATDC5 cells, PMCs, and PHCs, and was expressed in areas of cartilage degradation or absorption in vivo. Overexpression of Runx2 or Sox9 increased miR-381 expression in ATDC5 cells. miR-381 suppressed expression of collagen, type II, alpha 1, and enhanced expression of metalloproteinase-13 (MMP-13), but did not regulate NFKBIA and NKRF activity. Conclusion: miR-381 was highly expressed during chondrogenesis and in arthritic cartilage. It may contribute to absorption of the cartilage matrix by repressing type II collagen and inducing MMP-13.

Copyright $(2015$ S. Karger AG, Basel
\end{abstract}

\section{Introduction}

Osteoarthritis (OA) is characterized by degeneration of joint cartilage. The prevalence of radiographic $\mathrm{OA}$, diagnosed based on the inter-bone distance in the affected joint, ranges C. Hou and F. Meng contributed equally to the present study. 
from $19-28 \%$ in the knee and $7-27 \%$ in the hip [1]. However, the frequency of symptomatic $\mathrm{OA}$, defined by pain, aching, or stiffness is much less frequent. In general, symptomatic OA affects less than $10 \%$ of the population, although rates as high as $17 \%$ have been reported [1]. The risk factors for developing $\mathrm{OA}$ include increased age, female gender, race, and genetic predispositions [1]. The current treatments for OA can relieve symptoms like pain and tenderness, but cannot cure the disease or stop its progression. An incomplete understanding of the molecular mechanisms that regulate cartilage formation and degeneration has slowed the development of novel OA therapies.

The pathogenesis of OA is thought to have a major inflammatory component that drives cartilage degradation and inhibits cartilage formation. The proinflammatory cytokine, interleukin-1 $\beta$ (IL-1 $\beta$ ), leads to the upregulation of matrix metalloproteinase (MMP) enzymes, which degrade the extracellular cartilage matrix (ECM) [2]. The ECM is secreted by chondrocytes and contributes to the mechanical function of cartilage. MMP-13 in particular has the capacity to degrade a wide range of matrix molecules, and is thought to be one of the major effectors induced by IL-1 $\beta$ in OA $[3,4]$. Type II collagen is a major component of the ECM, and an MMP-13 target, that is degraded in osteoarthritic cartilage [5-7]. The transcription factor SRY-related high mobility group-box-9 (Sox9) initiates type II collagen expression, and inhibits the expression and activity of Runx2, a key regulator of collagen degradation that upregulates MMP-13 [8, 9].

While chondrogenic differentiation is regulated by many different factors, including retinoic acid receptor signaling, microRNAs (miRNAs), small non-coding RNAs that posttranscriptionally regulate gene expression, have been implicated in regulating all stages of ossification [10-13]. For example, miR-140 regulates cartilage and bone formation [14, 15], and miR-365 increases chondrocyte proliferation and differentiation [16]. miRNAs have also been implicated in the development of arthritic cartilage $[13,17]$. For example, IL-1 $\beta$ significantly represses miR-558, which relieves its inhibition of Cyclooxygenase-2 (Cox2) and increases cartilage catabolism [18]. Another example is miR-194, which inhibits expression of Sox 5 and thereby inhibits chondrogenic differentiation in adipose stem cells [19]. We were the first to describe an approximately 3-fold upregulation in miR-381 when human mesenchymal stem cells differentiate to chondrocytes [20]. We also identified chondrogenesis related genes that are putative targets of miR-381 including NFKBIA; CEBP $\beta$; Runx2; Sox-2, 4, 5, 6, and 9; SMAD-3, 4, and 5; MAPK-1 and 9; BMPR2, and MMP7 [20]. To date, there is very little known about miR-381. It has been most thoroughly characterized in cancer models where it interacts with cell cycle genes. miR-381 has been reported to act as an "oncomir" in glioma progression by enhancing proliferation of glioma cells, and to suppress lung adenocarcinoma by inhibiting cell migration and invasion [21-23]. Given the role of miRNAs in regulating cartilage homeostasis, and the putative chondrogenesis targets of miR-381, we hypothesized that miR-381 might have a role in OA pathogenesis. Here we investigated the regulation and molecular mechanism of action for miR-381 in chondrogenesis and cartilage degeneration.

\section{Materials and Methods}

\section{Primary chondrocyte isolation}

This study adhered to the standards of the Ethics Committee on Human Experimentation at the First Affiliated Hospital of Sun Yat-Sen University, China and the Declaration of Helsinki (2000). After obtaining informed consent, primary human chondrocytes (PHCs) were isolated from three hip or knee joints during amputation surgery to treat osteosarcoma at the First Affiliated Hospital of Sun Yat-Sen University. Patients with osteoarthritis and local or systemic immunological disorders were excluded based on the clinical diagnosis. In a second patient set, degraded joint cartilage was collected from OA hips $(\mathrm{n}=3)$ during total hip arthroplasty surgery. Cartilage was cut into pieces $1 \mathrm{~mm}$ in diameter, and digested with DMEM/F12 (Hyclone, Logan, UT, USA) supplemented with $2 \%$ penicillin and streptomycin (Gibco, Grand Island, NY, 
USA), 5\% FBS (Gibco, 12657), and 0.4\% Pronase (Roche, Indianapolis, IN, USA) for $90 \mathrm{~min}$. The tissue pieces were then transferred to a second digestion solution consisting of DMEM/12 (Hyclone) supplemented with $2 \%$ penicillin and streptomycin (Gibco), 5\% FBS (Gibco), and $0.025 \%$ Collagenase P (Roche) for $7 \mathrm{~h}$ on a $37^{\circ} \mathrm{C}$ stir-plate. The chondrocytes were seeded in flasks with DMEM/F12 plus 5\% FBS, 2\% penicillin, and streptomycin.

Mice were cared for in accordance with guidelines of Animal Center of the First Affiliated Hospital of Sun Yat-Sen University. Primary mouse chondrocytes (PMCs) were isolated as described previously [24]. Briefly, newborn mice ( 5 days old) were washed with $70 \%$ ethanol and sacrificed under general anesthesia. The cartilage was obtained from the femoral head, femoral condyle, and tibial plateau and digested with $3 \mathrm{mg} / \mathrm{mL}$ collagenase D in complete culture medium (M199 (Gibco 11150-059) supplemented with 10\% FBS and $1 \%$ penicillin and streptomycin) for 90 minutes at $37^{\circ} \mathrm{C}$ with agitation. Then the cartilage was digested again in collagenase $\mathrm{D}$ overnight at $37^{\circ} \mathrm{C}$. The cells were dislodged from the cartilage with agitation, filtered through a $48 \mathrm{um}$ nylon mesh, and then seeded in a culture flask at a density of 8,000 cells $/ \mathrm{cm}^{2}$. Early-passage PMCs, defined as no more than four passages, were used in these experiments. For a second set of experiments, pregnant C57BL/6J mice were purchased from the Animal Center of the First Affiliated Hospital of Sun Yat-Sen University. Embryos were collected on E10.5 and E14.5 and used to detect miR-381 expression in areas undergoing active cartilage absorption in the early limb buds.

\section{Cell culture}

ATDC5 mouse cells (Riken Cell Bank; Ibaraki, Japan) were cultured in DMEM/F12 supplemented with $5 \% \mathrm{FBS}$, and $1 \%$ penicillin and streptomycin at $37^{\circ} \mathrm{C}$ in a humidified $5 \% \mathrm{CO}_{2}$ atmosphere. The culture medium was changed every 2 days. The cells were split when they reached confluence. All of the experiments were completed using cells passaged fewer than 20 times.

\section{Inducing chondorogeneis in ATDC5, mouse, and human cells}

Chondrogenesis was induced in ATDC5 cells for 14 days. The chondrogenic culture medium was changed every day. PMCs were grown in M199 medium (Gibco, 11150-059) supplemented with 10\% FBS, 1\% penicillin and streptomycin, bFGF (Peprotech, Rocky Hill, NJ, USA), EGF (Peprotech), and insulin (Sigma, St. Louis, MO, USA) at $37^{\circ} \mathrm{C}$ in a humidified $5 \% \mathrm{CO}_{2}$ atmosphere. PHCs were cultured in DMEM/F12 supplemented with 5\% FBS, 1\% penicillin and streptomycin, and ITS (insulin, transferrin, and selenous acid) + Premix (BD Biosciences, San Jose, CA, USA catalog: 354352) as previously described [25-29] at $37^{\circ} \mathrm{C}$ in a humidified $5 \% \mathrm{CO}_{2}$ atmosphere. Chondrogenic ATDC5 cells, PHCs, and PMCs were treated with recombinant IL-1 $\beta$ (Peprotech, 200-01B) using various doses and times [30-32]. Where indicated, the cells were transfected with an mmu-miR-381 mimic, a synthetic miRNA with the same sequence as mature miR381 (micrON $^{\mathrm{TM}}$ mmu-miR-381-3p mimic, miR10000746-1-5), an miR-381 inhibitor, synthetic miRNA that interferes with miR-381 (micrOFF ${ }^{\mathrm{TM}}$ mmu-miR-381-3p inhibitor, miR20000746-1-5), or negative control miRNA, synthetic miRNA with a nonsensical sequence (micrON ${ }^{\mathrm{TM}}$ miRNA mimic control, miR01201-1-5; micrOFF ${ }^{\mathrm{TM}}$ miRNA inhibitor control, miR02201-1-5; Ribobio, Guangzhou, China).

\section{Alcian blue staining}

Cultured cells were fixed in formalin for 4 hours at room temperature and stained with alcian blue 8GX (Cyagen) for $20 \mathrm{~min}$ at room temperature.

\section{In situ hybridization}

Tissue from mouse embryos at days E10.5 and E14.5 were harvested and processed with human joint cartilage obtained from OA hip patients. The tissues were fixed in DEPC treated with 4\% paraformaldehyde at $4^{\circ} \mathrm{C}$ overnight and embedded in paraffin. $5 \mu \mathrm{m}$ sections were incubated with $40 \mu \mathrm{g} / \mathrm{mL}$ Proteinase $\mathrm{K}$ (Promega, Madison, WI, USA catalog: V3021) at room temperature overnight. The following day, the sections were incubated with $5^{\prime}$-labeled RNA probe (Exiqon, 38188 ) at $56^{\circ} \mathrm{C}$ overnight. Endogenous alkaline phosphatase was blocked at room temperature for $1 \mathrm{~h}$, followed by incubation with anti-Digoxigenin-AP (1:500, Roche, 11093274$)$ at $4^{\circ} \mathrm{C}$ overnight. The nitroblue tetrazolium (NBT)/BCIP (Sigma, N1911) staining reaction was then performed, the sections were counterstained with safrinin 0 and dehydrated with ethanol. Negative control staining was performed using PBS.

\section{KARGER}


RNA extraction, reverse transcription, and quantitative real-time reverse transcription-polymerase chain reaction ( $q R T-P C R)$

Total RNA was extracted with miRNeasy Mini Kit (QIAGEN, USA) following the manufacture's instruction. The concentration and purity of the extracted RNA was analyzed using the Epoch Multi-Volume Spectrophotometer System (BIOTEK, USA). cDNA was generated using the PrimeScript® miRNA cDNA Synthesis Kit (Takara, Japan) following the manufacture's instruction. qRT-PCR was performed using SYBR $\mathbb{R}$ Premix Ex Taq ${ }^{\text {TM }}$ II (Takara, Japan) and the BioRad IQ5 system according to the manufacturer's instruction. The specific primers are listed in Table 1. The reverse primer for miRNA-381 was the Uni-miR qPCR Primer (Takara, Japan, Cat: D352). The melting curve was used to monitor the quality of the pRT-PCR reactions. The fold change in mRNA expression was calculated using the $\Delta \Delta \mathrm{Ct}$ method. All samples were measured in triplicate.

\section{Transfection}

ATDC5 cells were cultured in DMEM/F12 supplemented with 5\% FBS until they reached $60 \%$ confluence. Lipofectamine ${ }^{\circledR} 2000$ Transfection Reagent (Invitrogen, 11668) was used to transfect cells according to the manufacturer's instruction. After 6 hours, the transfected ATDC5 cells were differentiated to chondrocytes using medium containing ITS+ Premix.

\section{Construction of eukaryotic expression plasmids}

Eukaryotic expression GV230 plasmids were purchased from Genechem (Shanghai, China). The plasmids were constructed using standard DNA techniques. Runx2 was cloned using the following primers: $5^{\prime}$-TAC CGG ACT CAG ATC TCG AGA TGG CGT CAA ACA GCC TCT TC-3', 5' - GAT CCC GGG CCC GCG GTA CCG TAT ATG GCC GCC AAA CAG ACT C-3'. Sox9 was closed using the primers: 5'-TCC GCT CGA GAT GAA TCT CCT GGA CCC CTT C-3', 5' -ATG GGG TAC CGT GGG TCT GGT GAG CTG TGT GTAG-3'. The amplified DNA was inserted into the $\mathrm{XhoI} / \mathrm{KpnI}$ restriction sites of GV230. The final plasmids were verified by sequencing.

Target prediction, construction of luciferase reporter vector, and luciferase reporter assay.

The potential binding sites of miR-381 were predicted using miRanda, miRDB, and TargetScan software. The DNA sequences containing predicted seed sequences of NFKBIA and NKRF $3{ }^{\prime}$-UTR were amplified by PCR using the primers: m-NFKBIA-3UTR-F: CCG ACT CGA GGT GGA AAG TGG CAA AAA G; m-NFKBIA-3UTR-R: ATT GCG GCC GCC TGT CTG TAA AAA TCT GTT; m-NKRF-F: CCG CCT CGA GGA TTT TAC TAA GTT GTC; and m-NKRF-R: ATT GCG GCC GCTT TTG ATC AAC TTT GG. The seed sequences were mutated using standard PCR techniques with the following primers: m-NFKBIA-mut-F:ATG TGG AGA ACA TAT TTT GTA CAA ATA GAG TT; m-NFKBIA-mut-R:GTA CAA AAT ATG TTC TCC ACA TTC TTT TTG C; m-NKRF-mut-F:CTG TCC AGA ACA TAA CTT AGC TTA ATC CAG CAG; and m-NKRF-mut-R: AAG CTA AGT TAT GTT CTG GAC AGA ATT CTC TAG.
Table 1. Primer sequences for qPCR

\begin{tabular}{lll}
\hline Gene & Primer sequences \\
\hline mmu-U6 & Forward & CTCGCTTCGGCAGCACA \\
& Reverse & AACGCTTCACGAATTTGCGT \\
mmu-GAPDH & Forward & TGTGTCCGTCGTGGATCTGA \\
& Reverse & TTGCTGTTGAAGTCGCAGGAG \\
mmu/hsa-mir-381 & & TATACAAGGGCAAGCTCTCTGT \\
mmu-col2a1 & Forward & CCCGCCTTCCCATTATTGAC \\
& Reverse & GGGAGGACGGTTGGGTATCA \\
mmu-Sox9 & Forward & GGGGGTGAGCTTTGATTAATTC \\
mmu-Col10a1 & Feverse & GGGATTTAAGGCTCAAGGTGTTT \\
& Forward & TTCTGCTGCTAATGTTCTTGACC \\
mmu-Runx2 & Feverse & GGGATGAAGTATTGTGTCTTGGG \\
& Forward & ATGCTTCATTCGCCTCACAAA \\
Reverse & GCACTCACTGACTCGGTTGG \\
mmu-mmp13 & Forward & ATGCATTCAGCTATCCTGGCCA \\
& Reverse & AAGATTGCATTTCTCGGAGCCTG \\
mmu-TNF-alpha & Forward & GACGTGGAACTGGCAGAAGAG \\
& Reverse & TTGGTGGTTTGTGAGTGTGAG \\
& Forward & TCCTGATGTGGGTGAATACAATG \\
& Reverse & GCCATCGTGAAGTCTGGTAAAAT \\
\hline
\end{tabular}


The amplified DNA sequences were inserted into pmiR-RB-REPORT ${ }^{\mathrm{TM}}$ Vector to generate wild type (WT) or mutated (MUT) NFKBIA/NKRF $3^{\prime}$-UTR luciferase vectors. To generate mutant NFKBIA and NKRF $3^{\prime}$-UTR vectors, we switched the predicted seed sequences CTTGTATA and CTTGTATT to GAACATAT and GAACATAA, respectively. All vectors were verified by sequencing.

For the reporter assay, $1.2 \times 10^{4}$ cells were cultured in a 96-well plates with $100 \mu \mathrm{L}$ of culture medium for $24 \mathrm{~h}$. The mmu-miR-381 mimic ( $5 \mathrm{pmol}$ ) and vector (100 ng) were cotransfected into each well. The transfected cells were cultured in fresh culture medium for an additional $48 \mathrm{~h}$. The luciferase reporter assay was carried out following the manufacturer's instructions (Dual-Glo® Luciferase Assay System, Promega). Luminescence was measured using the Veritas 9100-002 luminometer.

\section{Statistical analysis}

All of the experiments were performed at least three times. The results are expressed as the mean and standard deviation (mean \pm SD). Both parametric and non-parametric inferential statistics were utilized in this study depending on whether the data was normally distributed. The independent t-test and MannWhitney U test were used to compare between two groups as appropriate. For multiple group comparisons, the one-way ANOVA and Kruskal-Wallis tests were utilized. A $p$ value less than 0.05 was considered statistically significant. All analyses were performed using IBM SPSS Version 20 (SPSS Statistics V20, IBM Corporation, Somers, New York).

\section{Results}

miR-381 was highly expressed in vitro during late stage chondrogenesis in ATDC5 cells and IL-1 $\beta$ induced chondrocytes

We have previously shown that stem cells derived from human adipose tissue express miR-381 when they are induced to differentiate to chondrocytes in vitro [20]. Here, ATDC5 cells were induced to differentiate to chondrocytes in vitro using ITS + Premix. miR-381 was significantly upregulated in chondrogeneic ATDC5 cells compared to control cells at 14 (3.07 \pm 0.34 vs. $14.58 \pm 1.7 ; p<0.001), 21(2.23 \pm 0.23$ vs. $8.50 \pm 1.38 ; p=0.002)$, and 28 (2.86 \pm 0.26 vs. $240.60 \pm 11.41 ; p=0.001$ ) days (Fig. $1 \mathrm{~A}$ ). Expression of miR-381 generally increased over time in the ATDC5 cells, although a slight decrease was observed at day 20 (Fig. 1A). Chondrocyte differentiation was verified through significantly increased expression of the chondrogenic markers collagen, type II, alpha 1 (Col2a1), Sox9, Collagen, type X, alpha 1 (Col10a1), and Runx2 (Fig. 1A). Sox9 and Col2a1 expression were noticeably increased at day 14 while Runx2 and Col10a1 peaked late at day 28 (Fig. 1A). Representative images of alcian blue staining in ITS + premix treated ATDC5 cells from 0-35 days are shown in Fig. 1B. Cartilage staining became stronger with prolonged chondrogenesis, indicating the accumulation of matrix, and peaked after 14 days.

To establish whether miR-381 expression was regulated by inflammation, we assessed the expression of miR-381 in IL-1 $\beta$ treated chondrogenic ATDC5 cells and primary chondrocytes. In the PHCs, IL-1 $\beta$ significantly increased the expression of miR-381 $1.6 \pm$ 0.2 -fold $(p<0.001$; Fig. 2A). MMP-13 mRNA was undetectable in PHCs in the absence of IL-1 $\beta$ after 40 cycles, but became detectable $(0.00060 \pm 0.0001$-fold; $p<0.001)$ after $24 \mathrm{~h}$ of stimulation with IL-1 $\beta$ (data not shown). Similarly, in PMCs (Fig. 2B) and ATDC5 cells (Fig. 2C) miR-381 was significantly upregulated in response to IL-1 $\beta$ after $24 \mathrm{~h}$ in culture by $1.44 \pm 0.42$-fold ( $p=0.031$ ) and $14.28 \pm 0.93$-fold ( $p<0.001)$, respectively. Similar to the PHCs, in PMCs and ATDC5 cells MMP-13 was significantly upregulated by $7.76 \pm 2.20$-fold $(p<0.001)$ and $16.74 \pm 3.55$-fold $(p<0.001)$, respectively after $24 \mathrm{~h}$. In the ATDC5 cells, MMP-13 expression remained elevated $(19.06 \pm 2.23$-fold; $p<0.001)$ up to $7 \mathrm{~d}$ in culture. IL-1 $\beta$ treatment also increased the expression of TNF- $\alpha(31.5 \pm 5.62$-fold; $p<0.001)$, another inflammatory cytokine, in PMCs at $24 \mathrm{~h}$. In ATDC5 cells, Col2a1 expression was significantly reduced after $24 \mathrm{~h}$ with IL-1 $\beta(0.81 \pm 0.08$-fold; $p=0.002)$ and was further reduced at 7 $\mathrm{d}(0.30 \pm 0.08$-fold; $p<0.001)$. Interestingly, across the in vitro models, expression of miR381, MMP-13, and Col2a1 expression were altered in response to IL-1 $\beta$ in a time dependent 


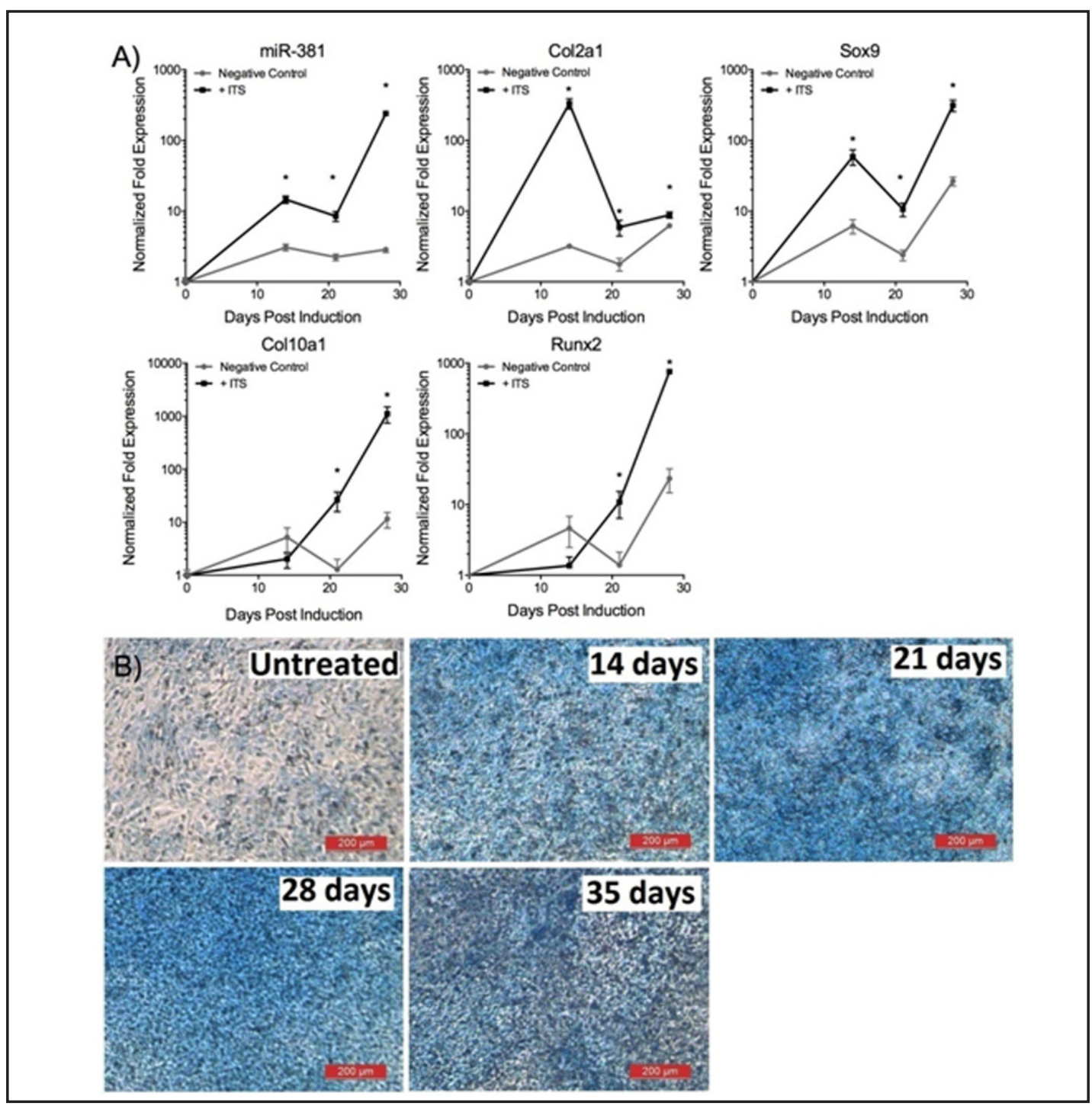

Fig. 1. Elevated expression of miR-381 during chondrogenesis. ATDC5 cells were induced to chondrogenesisby incubating withmedia containing insulin, transferrin, and selenous acid (ITS) + Premix for the indicated amount of time. (A) Gene expression and collagen deposition in treated cells was compared to untreated cells by qPCR. The expression levels of miR-381,chondrogenic markers(Col2a1 and Sox9) and hypertrophic markers (Col10a1and Runx2) were measured. Untreated ATDC5 cells (F) or ATDC5 cells treated for 14 (G), $21(\mathrm{H}), 28$ (I), or 35 days (J) were fixed with formalin and stained with alcian blue to detect collagen deposition. ${ }^{*} \mathrm{p}<0.05$.The data are representative of an average of 6 independent assays, each with 3 samples per group.

manner. Taken together, these results suggested that inflammation might contribute to regulating miR-381 mediated chondrogenesis.

miR-381 is expressed in in vivo models of late stage of chondrogenesis and arthritis

To confirm our in vitro findings in a more physiologically relevant in vivo model, we stained mouse embryos and arthritic cartilage from OA patients by in situ hybridization to determine whether miR-381 was present. The negative control and Safrinin O counterstain for each representative image is shown. miR-381 was observed in the early cartilage tissues of the embryonic mouse limb bud, especially in the areas of the toe webs that were being absorbed (Fig. 3A). miR-381 was also evident in areas of endochondral ossification in the toe 


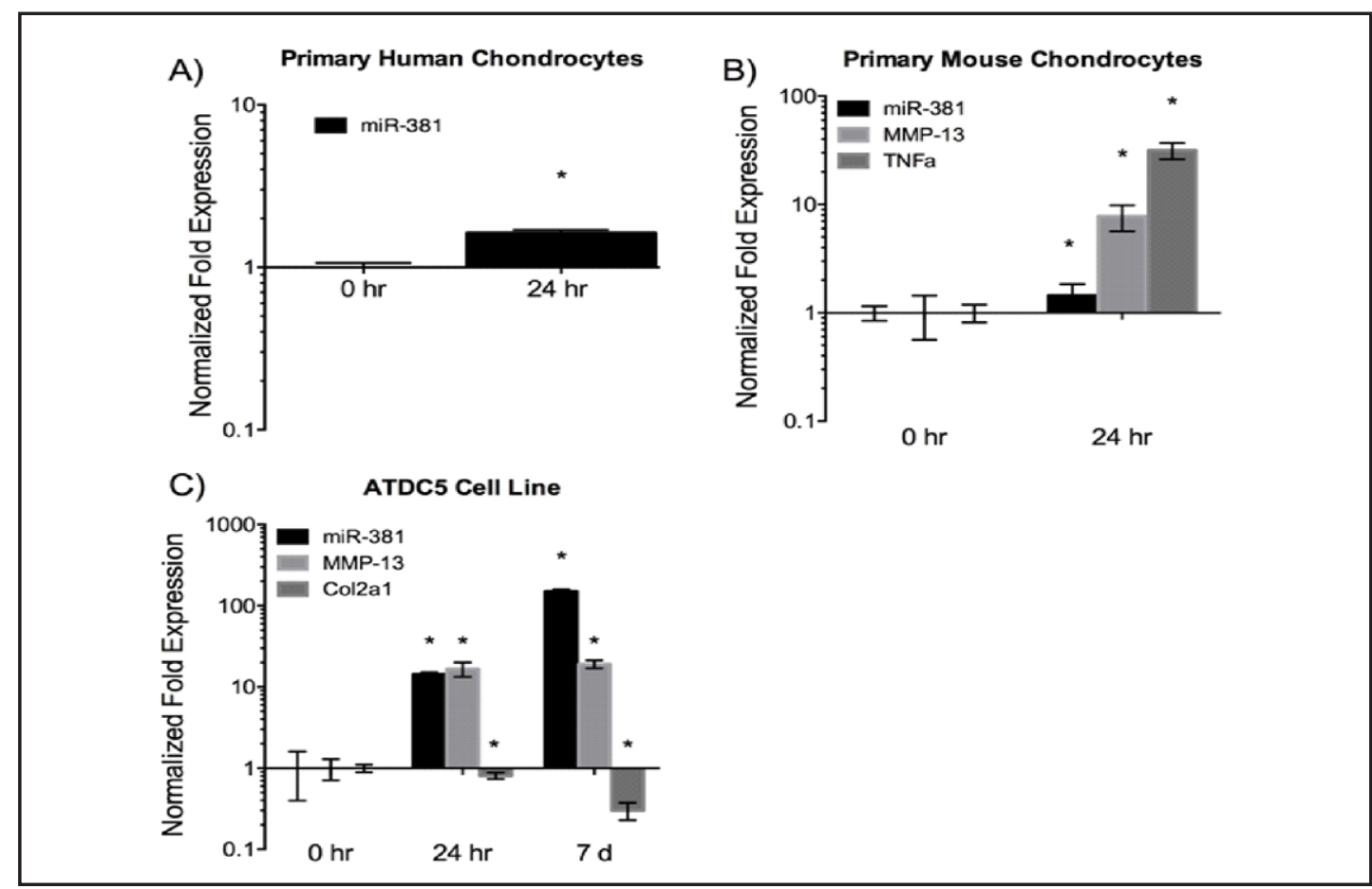

Fig. 2. miR-381 expression is elevated in chondrocytes following treatment with IL-1ß.Primary human chondrocytes (PHCs; A) and primary mouse chondrocytes (PMCs; B) were treated with $1 \mathrm{ng} / \mathrm{mL}$ of IL- $1 \beta$ for $4 \mathrm{~h}$. ATDC5 cells were induced to become chondrocytes insulin, transferrin, and selenous acid (ITS) + Premix for 14 days prior to treatment with $1 \mathrm{ng} / \mathrm{mL}$ IL-1 $\beta$ for the indicated amount of time (C). The expression levels of miR-381, TNF- $\alpha$, MMP-13, and Col2a1 expression were measured by qPCR and shown in fold induction relative to unstimulated cells. ${ }^{*} \mathrm{p}<0.05$. The data are representative of an average of 3 independent assays, each with 5 samples per group.

Fig. 3. miR-381 is evident in mouse embryos and cartilage from osteoarthritic patients. miR-381expression in vivo was assessed using in situ hybridization with nitroblue tetrazolium (NBT)/BCIP-labeled probes (blue). Tissues were counterstained with nuclear Safrinin O (red). Representative staining is shown for miR-381 in limb buds (A) and claws (B) of E10.5 mouse embryos. Endochondral ossification is highlighted in the toe webs and metacar-

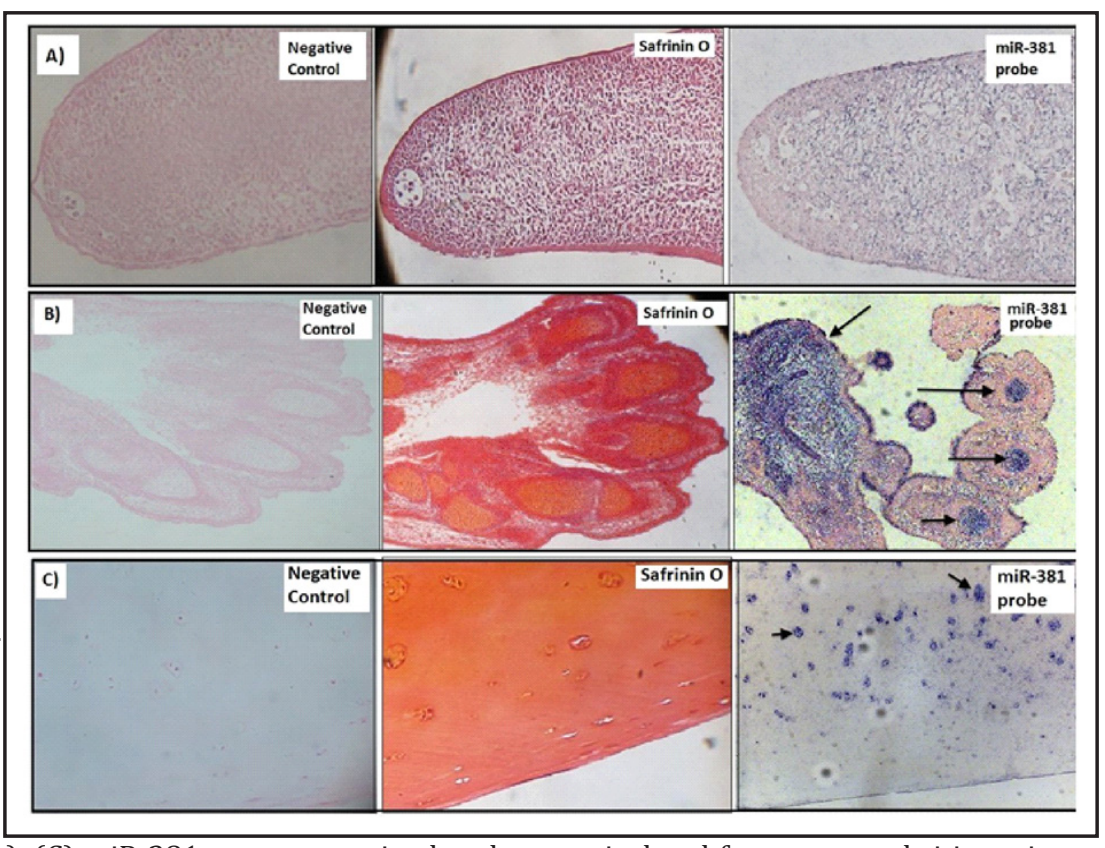
pal bones (black arrows). (C) miR-381 was present in chondrocytes isolated from osteoarthritic patients. The cartilage was cut and spread to provide a full depth view, and the bottom of the photo was the cartilage surface. Black arrows designate strongly stained chondrocytes in deeper layers of the cartilage. 


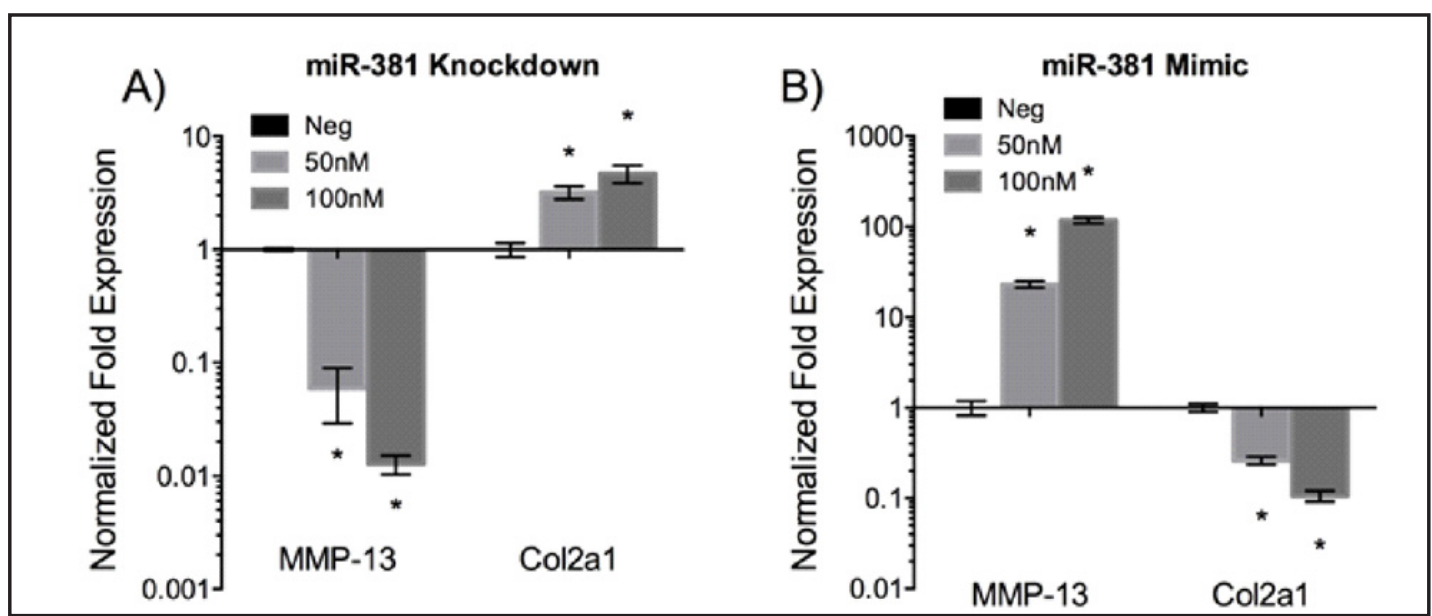

Fig. 4. Effect of miR-381 on Col2a1 and MMP-13. ATDC5 cells transfected with a synthetic RNA miR-381 inhibitor (A) or synthetic RNA mimic(B) were treated with insulin, transferrin, and selenous acid (ITS) + Premix to induce chondrogenesis. ATDC5 cells were treated with a non-sense synthetic RNA as the negative control. The expression levels of MMP-13 and Col2a1 were determined with $\mathrm{qPCR} .{ }^{*} \mathrm{p}<0.05$.The data are representative of an average of 3 independent assays, each with 6 samples per group.

and metacarpal bones (Fig. 3B). In the OA patients, miR-381 was expressed in chondrocytes from joint cartilage (Fig. 3C). The anti-miR-381 probe stained chondrocytes in the deep layer of the articular cartilage, which are primarily hypertrophic chondrocytes [33-35]. These data suggested that miR-381 is involved in late stage chondrogenesis and endochondral ossification, and is present in osteoarthritic joints in vivo.

miR-381 repressed type II collagen expression and enhanced MMP-13 expression

To establish a mechanistic link between miR-381 and chondrogenesis and cartilage degradation, we either inhibited miR-381 or over expressed an miR-381 mimic in ATDC5 cells. The ATDC5 cells were induced to differentiate into chondrocytes and transfected with either the inhibitor for 7 days (Fig. 4A) or the mimic for 3 days (Fig. 4B). The expression of MMP-13 and Col2a1 was assessed by qRT-PCR and compared to negative control ATDC5 cells. The miR-381 mimic induced a dose dependent decrease in the expression of Col2a1 (50nM: $0.26 \pm 0.06$-fold, $p=0.004 ; 100 \mathrm{nM}$ : $0.11 \pm 0.04$-fold, $p=0.003$ ), and a dose dependent increase in the expression of MMP-13 (50nM: $23.04 \pm 4.37$-fold, $p=0.019 ; 100 \mathrm{nM}: 117.82 \pm$ 19.43 -fold, $p<0.001$ ). In contrast, the miR-381 inhibitor induced a dose dependent increase in Col2a1 expression (50nM: $3.19 \pm 0.32$-fold, $p=0.008$; $100 \mathrm{nM}$ : $4.69 \pm 2.05$-fold, $p=0.02$ ) and dose dependent decrease in MMP-13 expression (50nM: $0.06 \pm 0.07$-fold, $p<0.001$; $100 \mathrm{nM}$ : $0.01 \pm 0.01$-fold, $p<0.001$ ). Neither the miR-381 mimic nor the inhibitor affected expression of Sox9, Runx2, or Col10a1 (data not shown).

\section{Runx2 and Sox9 upregulated expression of miR-381}

Given the roles of Sox9 and Runx2 in regulating Col2a1 and MMP-13 expression, we next investigated whether Runx2 and Sox 9 also regulated miR-381 expression during ATDC5 chondrogenesis. The expression levels of Runx2 and Sox9 were increased or decreased using targeted siRNA or plasmids that overexpressed Runx2 (Fig. 5A) or Sox9 (Fig. 5B). Targeted siRNA significantly reduced the expression of Runx2 $(0.77 \pm 0.13$-fold, $p<0.05)$ and significantly reduced the expression of miR-381 ( $0.17 \pm 0.03$-fold, $p<0.05$; Fig. $5 \mathrm{~A})$. However, transfecting a plasmid containing the Runx2 gene significantly increased expression of both Runx2 (1.95 \pm 0.64 -fold, $p<0.01)$ and miR-381 (2.79 \pm 0.75 -fold, $p<0.05)$. Similar results were observed for Sox9, although the reduction in miR-381 expression when Sox 9 was knocked down was not significant (Fig. 5B). Thus, its likely that both Runx2 and Sox 9 contributed to regulating the expression of miR-381 in vitro.

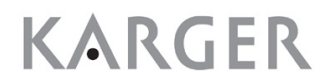




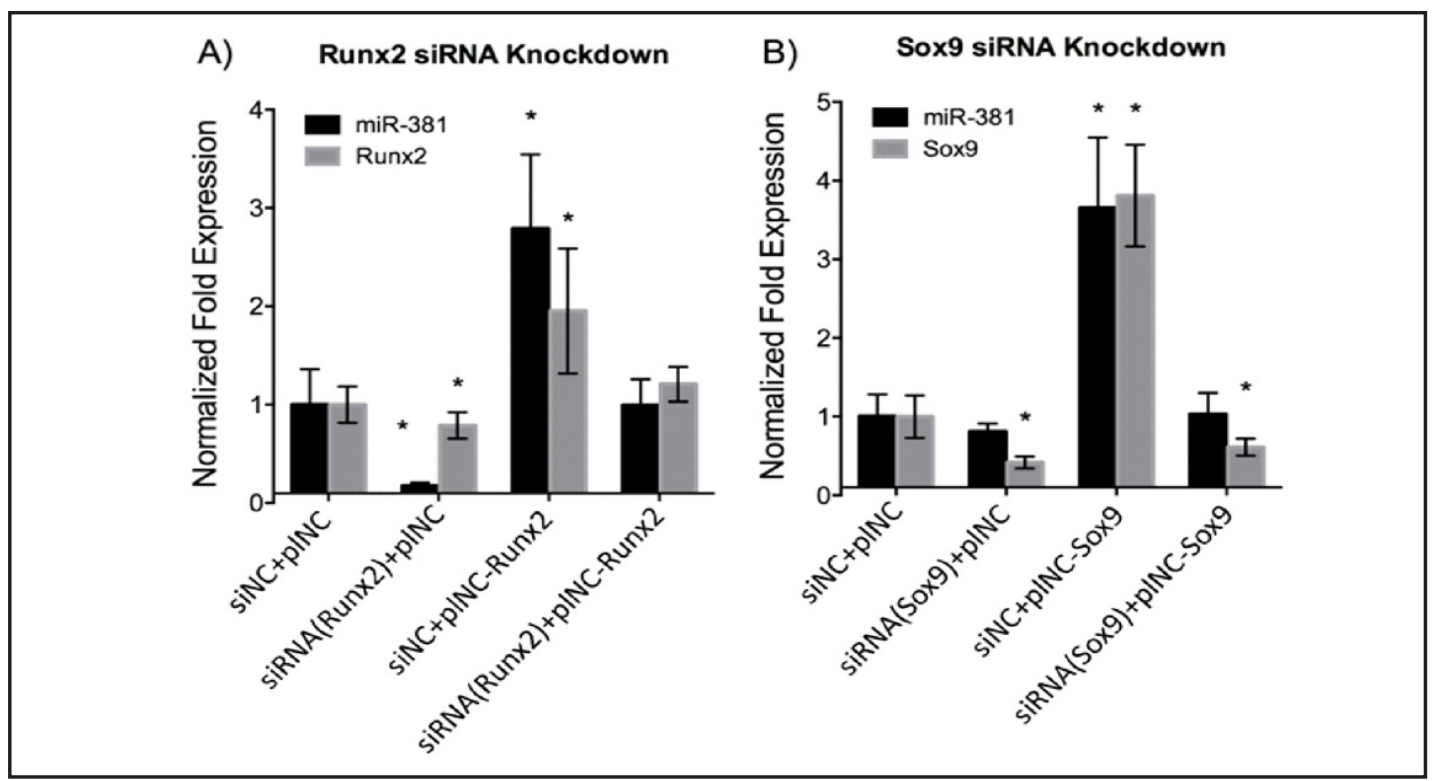

Fig. 5. Regulation of miR-381 by Runx2 and Sox9. ATDC5 cells were cotransfected with negative control siRNA (siNC), Runx2 (A) or Sox9 (B) siRNA and empty vector (GV230; plNC) or a GV230 plasmid containing Runx2 (A) or Sox9 (B). Chondrogenesis of ATDC5 was induced using insulin, transferrin, and selenous acid (ITS) + Premix and the expression levels of miR-381, Sox9, and Runx2 were measured with qPCR. * p $<0.05$. The data are representative of an average of 4 independent assays, each with 6 samples per group.

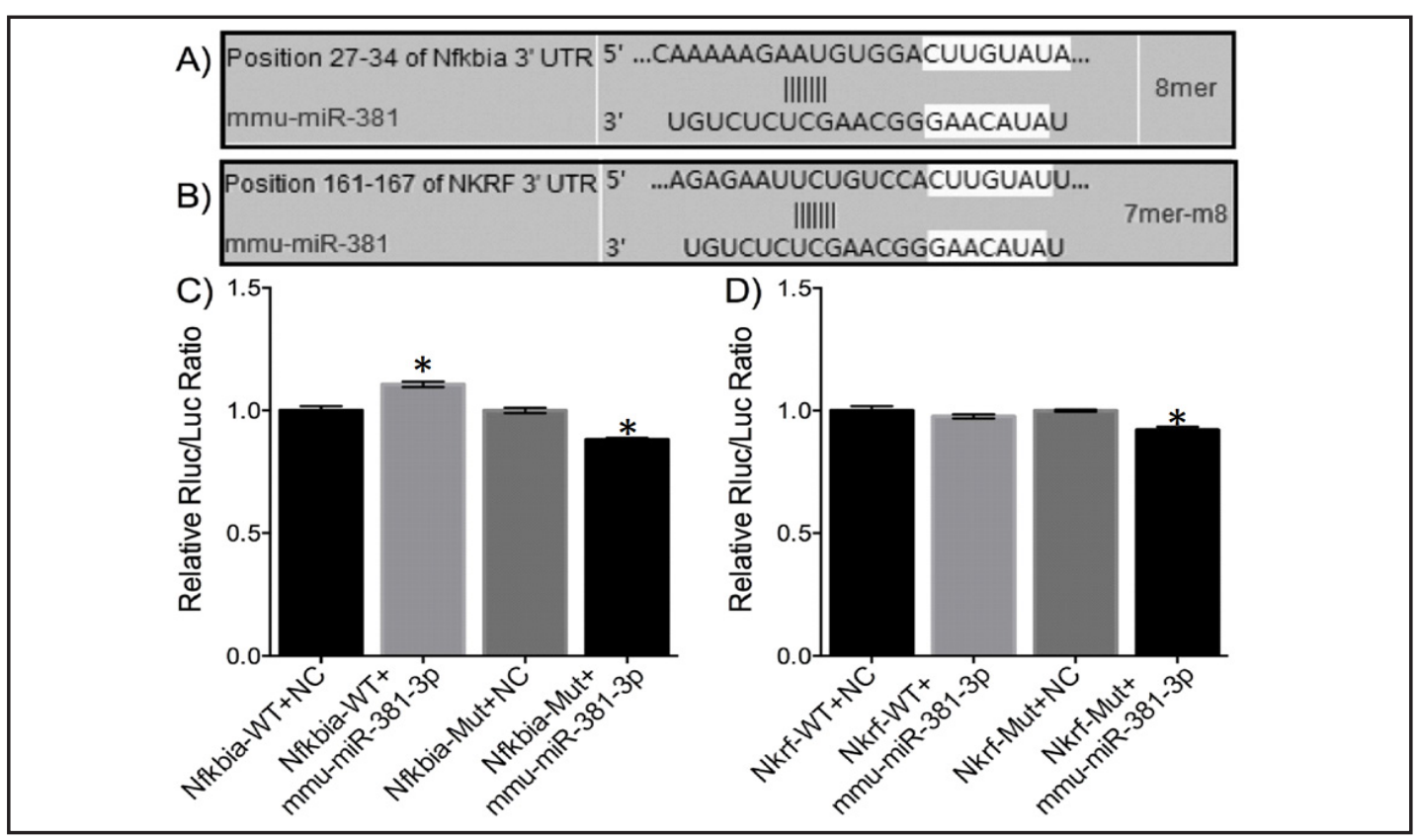

Fig. 6. NFKBIA and NKRF regulation by miR-381. (A) Wild type $3^{\prime}$-UTR and mutant of NFKBIA (Nfkbia-WT; Nfkbia-Mut) or (B) Wild-type and mutant 3'-UTR of NKRF (NKRF-WT; NKRF-Mut) were inserted into luciferase reporter vectors. The constructed vectors and miR-381 mimic (mmu-miR-381-3p) or non-sense negative control (NC) were cotransfected into cells. The fluorescence intensity was measured. $* p<0.05$. The data are representative of an average of 4 independent assays, each with 3 samples per group.

miR-381 does not target NFKBIA or NKRF

NF-kB has been reported to enhance MMP-13 expression and repress type II collagen expression [36, 37]. Based on our previous work [20], we hypothesized that miR-381 might 
affect the expression of nuclear factor of kappa light polypeptide gene enhancer in B-cells inhibitor, alpha (NFKBIA) and NFKB repressing factor (NKRF), which are NF-kB inhibitors [38]. The putative miR-381 binding sites for NFKBIA (Fig. 6A) and NKRF (Fig. 6B) are shown. Luciferase reporter assays with the wildtype or mutant $3^{\prime}$ UTR of NFKBIA and NKRF were performed in the presence or absence of the overexpressed miR-381 mimic. The mutant $3^{\prime}$ UTR sequence prevented the binding of the miR-381 mimic. Transfection of the miR-381 mimic slightly increased transcription from the wildtype $3^{\prime}$ UTR of NFKBIA $(1.1 \pm 0.02$-fold, $p=0.006$ ), suggesting that NFKBIA is not a target of miR-381 mediated repression (Fig. 6C). Surprisingly, when the NFKBIA seed sequence was mutated, the miR-381 mimic slightly reduced luciferase expression from the mutant $3^{\prime}$ UTR ( $0.88 \pm 0.01$-fold, $\left.p=0.0008\right)$, however this was not considered to be biologically relevant as the mutant seed sequence is not found in vivo. Similar results were observed from NKRF (Fig. 6D), indicating that neither NF-kB inhibitors were likely to be targets for miR-381 mediated repression.

\section{Discussion}

We hypothesized that miR-381 might play a role in cartilage degeneration and chondrogenesis. To test this hypothesis, we used in vitro models (PHCs, PMCs, and ATDC5 cells) and examined miR-381 expression in two different in vivo models of cartilage degeneration/ adsorption, cartilage from arthritic joints and from mouse limb buds during embryogenesis. We demonstrated that miR-381 was expressed in the in vitro systems during differentiation to chondrocytes and in vivo in areas of active cartilage degeneration. Furthermore, miR-381 expression is induced by IL-1 $\beta$, which is consistent with the inflammatory milieu commonly seen in OA [39], and regulated by Runx 2 and Sox9. Finally, miR-381 increased the expression of MMP-13 and decreased Col2a1 expression. Together these data suggest that miR-381 plays an active role in regulating cartilage degeneration.

Kawai et al. proposed that condrogenesis in ATDC5 cells progresses in stages based on the kinetics of TGF- $\beta 2$ and fibronectin expression [40]. He proposed that TGF- $\beta 2$ and fibronectin were active during the early (7-12 days) stages of chondrogenesis. In contrast, we observed that miR-381 expression was elevated particularly during late-stage (after day 28 in ATDC5 cells) of endochondral ossification. Sox9 and Runx2 play central roles in the earlyand late-stages of endochondral ossification. Runx2 has also been reported to participate in cartilage degradation and ossification under inflammatory conditions [41]. Regarding the slight decrease in miR-381, Col2a1, and Sox9 expression levels at day 21, the decrease and subsequent rebound are consistent with the expression patterns of proteoglycan in previous studies [42, 43], and likely reflects an intrinsic characteristic of ATDC5 cells as they transition from the early to late stage of chondrogenesis. Our data suggested that the expression levels of Sox 9 and Runx 2 are positively correlated with transcription of miR-381, indicating they may regulate expression of miR-381. The mechanism of regulation remains to be fully elucidated and warrants further study.

MMP-13 is a key regulator of OA and collagen degradation that has been shown to participate in late-stage endochondral ossification $[8,44]$. It is induced by CEBP $\beta$ and Runx2, both putative targets of miR-381 [9], and constitutive activation of Runx2 enhances MMP-13 expression [45-48]. miR-381 increased the expression of MMP-13 in ATDC5 chondrocytes and was associated with reduced collagen deposition and Col2a1 expression. MMP-13 has been shown to be regulated by other miRNAs including miR-27a, miR-27b, miR-146, and miR-9 [49-52]. In the case of miR-27b, the regulation is direct through the interaction of the miRNA and the MMP-13 3' UTR [49]. However, miRNA regulation can also be indirect as in the case of miR-146, which regulated MMP-13 through Irak1 and Traf6 [50]. Our data suggest that miR-381 may be a signaling intermediate between Runx2 and MMP-13 during cartilage degradation. In the context of inflammation, as would be expected in OA patients, IL-1 $\beta$ stimulation increased the expression of MMP-13 in PHCs, PMCs, and ATDC5 cells, and 
concurrently upregulated miR-381 in all three systems. These data strongly suggest a role for miR-381 in OA pathogenesis via MMP-13 regulation.

The NF-kB pathway has also been reported to enhance MMP-13 expression and repress type II collagen expression $[36,53]$. Two key inhibitory components of the NF-kB signaling pathway, NFKBIA and NKRF, were predicted to be targets of miR-381. However, neither NFKBIA nor NKRF were targeted by miR-381 in chondrogenesis. A comprehensive literature search did not identify any published studies that have used agomir/antagomir, or similar RNA-based, strategies in clinical trials or pre-clinical animal models to treat OA. In vitro work has suggested that other promising candidates for RNA-based OA therapy include miR-140, miR-27a/b, and miR-22 [30, 49, 51, 54]. However, MMP-13 inhibitors have already been developed to treat OA [55] and targeting miR-381 may be another viable therapeutic strategy to treat OA.

In this study, we are the first to characterize the role of miR-381 in chondrogenesis and cartilage degeneration. The mechanistic insights we were able to offer into miR-381 function in chondrocytes were limited, as the luciferase assay did not conclusively show that miR-381 bound to the 3' UTR of NFKBIA and NKFR. This leaves open the possibility that miR-381 acts on chondrocytes through other mechanisms. Overall, our data suggest that miR-381 is a viable therapeutic target for preventing cartilage degradation. Therapeutic strategies incorporating microRNA have been applied in several disease states, including cancer, diabetes, and cardiovascular disease [56-58]. miR-155 has also been targeted recently as a therapeutic in inflammatory diseases including rheumatoid arthritis [59]. The strategies fall broadly into two categories: (1) inhibitory anti-sense RNA and (2) exogenous miRNA like molecules [56]. In the case of miR-381, an inhibitory anti-sense RNA therapeutic could be a candidate for OA treatment.

\section{Disclosure Statement}

None of the authors have a potential conflict of interest or the appearance of a conflict of interest with regard to the work.

\section{Acknowledgement}

We thank Xuerong Li, Shan Li, and Shang Mei in the Department of Parasitology, Zhongshan School of Medicine, Sun Yat-sen University, Guangzhou 510080, People's Republic of China for their aid with the microscopy studies and other necessary facilities for the present study. We offer special thanks to the Guangzhou Elite Project, which supported the work that was finished at Washington University in Saint Louis.

This study was supported by the National Natural Science Foundation of China (81301558, 81171709, 81201388 and 81371941), Doctoral Scientific Fund Project of the Ministry of Education of China (20130171120074), Basic scientific training project for young medical teachers of Sun Yat-sen University (15ykpy11) Natural Science Foundation of Guangdong Province, China (2013040016269). The study sponsors were not involved in the study design; data collection, analysis and interpretation; writing of the manuscript; or in the decision to submit the manuscript for publication. We offer special thanks to the Guangzhou Elite Project (Suijing [2014] 7, JY201420), which supported the work that was finished at Washington University in Saint Louis.

\section{References}

Zhang Y, Jordan JM: Epidemiology of Osteoarthritis. Clin Geriatr Med 2010;26:355-369.

Le LT, Swingler TE, Clark IM: Review: the role of microRNAs in osteoarthritis and chondrogenesis. Arthritis Rheum 2013;65:1963-1974.

-3 Shlopov BV, Gumanovskaya ML, Hasty KA: Autocrine regulation of collagenase 3 (matrix metalloproteinase 13) during osteoarthritis. Arthritis Rheum 2000;43:195-205. 


\section{Cellular Physiology Cell Physiol Biochem 2015;36:1753-1766 \begin{tabular}{l|l|l}
\hline DOI: $10.1159 / 000430148$ & C 2015 S. Karger AG, Basel
\end{tabular}

Hou et al.: MiR-381 in Chondrogenesis and Chondrocytes

4 Huebner JL, Otterness IG, Freund EM, Caterson B, Kraus VB: Collagenase 1 and collagenase 3 expression in a guinea pig model of osteoarthritis. Arthritis Rheum 1998;41:877-890.

5 Stoop R, van der Kraan PM, Buma P, Hollander AP, Billinghurst RC, Poole AR, van den Berg WB: Type II collagen degradation in spontaneous osteoarthritis in C57Bl/6 and BALB/c mice. Arthritis Rheum 1999;42:2381-2389.

-6 Stoop R, van der Kraan PM, Buma P, Hollander AP, Poole AR, van den Berg WB: Denaturation of type II collagen in articular cartilage in experimental murine arthritis. Evidence for collagen degradation in both reversible and irreversible cartilage damage. J Pathol 1999;188:329-337.

7 Hollander AP, Heathfield TF, Webber C, Iwata Y, Bourne R, Rorabeck C, Poole AR: Increased damage to type II collagen in osteoarthritic articular cartilage detected by a new immunoassay. J Clin Invest 1994;93:17221732.

8 Nishimura R, Wakabayashi M, Hata K, Matsubara T, Honma S, Wakisaka S, Kiyonari H, Shioi G, Yamaguchi A, Tsumaki N, Akiyama H, Yoneda T: Osterix regulates calcification and degradation of chondrogenic matrices through matrix metalloproteinase 13 (MMP13) expression in association with transcription factor Runx2 during endochondral ossification. J Biol Chem 2012;287:33179-33190.

-9 Hirata M, Kugimiya F, Fukai A, Saito T, Yano F, Ikeda T, Mabuchi A, Sapkota BR, Akune T, Nishida N, Yoshimura N, Nakagawa T, Tokunaga K, Nakamura K, Chung UI, Kawaguchi H: C/EBPbeta and RUNX2 cooperate to degrade cartilage with MMP-13 as the target and HIF-2alpha as the inducer in chondrocytes. Hum Mol Genet 2012;21:1111-1123.

10 Diederichs S, Zachert K, Raiss P, Richter W: Regulating chondrogenesis of human mesenchymal stromal cells with retinoic acid receptor-beta inhibitor: differential sensitivity of chondral verus osteochondral development. Cell Physiol Biochem 2014;33:1607-1619.

-11 Kobayashi T, Lu J, Cobb BS, Rodda SJ, McMahon AP, Schipani E, Merkenschlager M, Kronenberg HM: Dicerdependent pathways regulate chondrocyte proliferation and differentiation. Proc Natl Acad Sci U S A 2008;105:1949-1954.

12 Dong S, Yang B, Guo H, Kang F: MicroRNAs regulate osteogenesis and chondrogenesis. Biochem Biophys Res Commun 2012;418:587-591.

13 Hong E, Reddi AH: MicroRNAs in chondrogenesis, articular cartilage, and osteoarthritis: implications for tissue engineering. Tissue Eng Part B Rev 2012;18:445-453.

14 Tuddenham L, Wheeler G, Ntounia-Fousara S, Waters J, Hajihosseini MK, Clark I, Dalmay T: The cartilage specific microRNA-140 targets histone deacetylase 4 in mouse cells. FEBS Lett 2006;580:4214-4217.

15 Yang J, Qin S, Yi C, Ma G, Zhu H, Zhou W, Xiong Y, Zhu X, Wang Y, He L, Guo X: MiR-140 is co-expressed with Wwp2-C transcript and activated by Sox 9 to target Sp1 in maintaining the chondrocyte proliferation. FEBS Lett 2011;585:2992-2997.

16 Guan YJ, Yang X, Wei L, Chen Q: MiR-365: a mechanosensitive microRNA stimulates chondrocyte differentiation through targeting histone deacetylase 4. FASEB J 2011;25:4457-4466.

17 Barter MJ, Bui C, Young DA: Epigenetic mechanisms in cartilage and osteoarthritis: DNA methylation, histone modifications and microRNAs. Osteoarthritis Cartilage 2012;20:339-349.

18 Park SJ, Cheon EJ, Kim HA: MicroRNA-558 regulates the expression of cyclooxygenase-2 and IL-1betainduced catabolic effects in human articular chondrocytes. Osteoarthritis Cartilage 2013;21:981-989.

19 Xu J, Kang Y, Liao WM, Yu L: MiR-194 regulates chondrogenic differentiation of human adipose-derived stem cells by targeting Sox5. PLoS One 2012;7:e31861.

20 Zhang Z, Kang Y, Zhang Z, Zhang H, Duan X, Liu J, Li X, Liao W: (2012) Expression of microRNAs during chondrogenesis of human adipose-derived stem cells. Osteoarthritis Cartilage 2012;20:1638-1646.

21 Rothschild SI, Tschan MP, Jaggi R, Fey MF, Gugger M, Gautschi O: MicroRNA-381 represses ID1 and is deregulated in lung adenocarcinoma. J Thorac Oncol 2012;7:1069-1077.

22 Tang H, Liu X, Wang Z, She X, Zeng X, Deng M, Liao Q, Guo X, Wang R, Li X, Zeng F, Wu M, Li G: Interaction of hsa-miR-381 and glioma suppressor LRRC4 is involved in glioma growth. Brain Res 2011;1390:21-32.

23 Hu Z, Shu Y, Chen Y, Chen J, Dong J, Liu Y, Pan S, Xu L, Xu J, Wang Y, Dai J, Ma H, Jin G, Shen H: Genetic polymorphisms in the precursor MicroRNA flanking region and non-small cell lung cancer survival. Am J Respir Crit Care Med 2011;,183:641-648.

24 Thirion S, Berenbaum F: Culture and phenotyping of chondrocytes in primary culture. Methods Mol Med 2004;100:1-14. 


\section{Cellular Physiology Cell Physiol Biochem 2015;36:1753-1766 \begin{tabular}{l|l|l} 
and Biochemistry & $\begin{array}{l}\text { DOI: 10.1159/000430148 } \\
\text { Publisned onlne: July 13, } 2015\end{array}$ & $\begin{array}{l}\text { O) 2015 S. Karger AG, Basel } \\
\text { www.karger.com/cpb }\end{array}$ \\
\cline { 2 - 3 }
\end{tabular}}

Hou et al.: MiR-381 in Chondrogenesis and Chondrocytes

25 Newton PT, Staines KA, Spevak L, Boskey AL, Teixeira CC, Macrae VE, Canfield AE, Farquharson C: Chondrogenic ATDC5 cells: an optimised model for rapid and physiological matrix mineralisation. Int J Mol Med 2012;30:1187-1193.

26 Liu X, Liu J, Kang N, Yan L, Wang Q, Fu X, Zhang Y, Xiao R, Cao Y: Role of insulin-transferrin-selenium in auricular chondrocyte proliferation and engineered cartilage formation in vitro. Int J Mol Sci 2014;15:1525-1537.

-27 Zhang L, Song H, Zhao X: Optimum combination of insulin-transferrin-selenium and fetal bovine serum for culture of rabbit articular chondrocytes in three-dimensional alginate scaffolds. Int J Cell Biol 2009;2009:747016.

28 Yao Y, Wang Y: ATDC5: an excellent in vitro model cell line for skeletal development. J Cell Biochem 2013;114:1223-1229.

29 Atsumi T, Miwa Y, Kimata K, Ikawa Y: A chondrogenic cell line derived from a differentiating culture of AT805 teratocarcinoma cells. Cell Differ Dev 1990;30:109-116.

- 30 Miyaki S, Nakasa T, Otsuki S, Grogan SP, Higashiyama R, Inoue A, Kato Y, Sato T, Lotz MK, Asahara H: MicroRNA-140 is expressed in differentiated human articular chondrocytes and modulates interleukin-1 responses. Arthritis Rheum 2009;60:2723-2730.

-31 Simsa-Maziel S, Monsonego-Ornan E: Interleukin-1beta promotes proliferation and inhibits differentiation of chondrocytes through a mechanism involving down-regulation of FGFR-3 and p21. Endocrinology 2012;153:2296-2310.

-32 MacRae VE, Farquharson C, Ahmed SF: The restricted potential for recovery of growth plate chondrogenesis and longitudinal bone growth following exposure to pro-inflammatory cytokines. J Endocrinol 2006;189:319-328.

-33 Chen-An P, Andreassen KV, Henriksen K, Karsdal MA, Bay-Jensen AC: Investigation of chondrocyte hypertrophy and cartilage calcification in a full-depth articular cartilage explants model. Rheumatol Int 2013;33:401-411.

34 Pullig O, Weseloh G, Ronneberger D, Käkönen S, Swoboda B: Chondrocyte differentiation in human osteoarthritis: expression of osteocalcin in normal and osteoarthritic cartilage and bone. Calcif Tissue Int 2000;67:230-240.

-35 Girkontaite I, Frischholz S, Lammi P, Wagner K, Swoboda B, Aigner T, Von der Mark K: Immunolocalization of type X collagen in normal fetal and adult osteoarthritic cartilage with monoclonal antibodies. Matrix Biol 1996;15:231-238.

-36 Goldring MB, Otero M, Tsuchimochi K, Ijiri K, Li Y: Defining the roles of inflammatory and anabolic cytokines in cartilage metabolism. Ann Rheum Dis 2008;67:S iii75-82.

37 Marcu KB, Otero M, Olivotto E, Borzi RM, Goldring MB: NF-kappaB signaling: multiple angles to target OA. Curr Drug Targets 2010;11:599-613.

38 Okamoto H, Cujec TP, Yamanaka H, Kamatani N: Molecular aspects of rheumatoid arthritis: role of transcription factors. FEBS J 2008;275:4463-4470.

-39 Goldring MB, Otero M, Plumb DA, Dragomir C, Favero M, El Hachem K, Hashimoto K, Roach HI, Olivotto E, Borzì RM, Marcu KB: Roles of Inflammatory and anabolic cytokines in cartilage metabolism: signals and multiple effectors converge upon MMP-13 regulation in osteoarthritis. Eur Cell Mater 2011;21:202-220.

40 Kawai J, Akiyama H, Shigeno C, Ito H, Konishi J, Nakamura T: Effects of transforming growth factor-beta signaling on chondrogenesis in mouse chondrogenic EC cells, ATDC5. Eur J Cell Biol 1999;78:707-714.

41 Sonomoto K, Yamaoka K, Oshita K, Fukuyo S, Zhang X, Nakano K, Okada Y, Tanaka Y: Interleukin-1beta induces differentiation of human mesenchymal stem cells into osteoblasts via the Wnt-5a/receptor tyrosine kinase-like orphan receptor 2 pathway. Arthritis Rheum 2012;64:3355-3363.

-42 Han F, Gilbert JR, Harrison G, Adams CS, Freeman T, Tao Z, Zaka R, Liang H,Williams C, Tuan RS, Norton PA, Hickok NJ: Transforming growth factor-beta1 regulates fibronectin isoform expression and splicing factor SRp40 expression during ATDC5 chondrogenic maturation. Exp Cell Res 2007;313:1518-1532.

43 Oca P, Zaka R, Dion AS, Freeman TA, Williams CJ: Phosphate and calcium are required for TGFbetamediated stimulation of ANK expression and function during chondrogenesis. J Cell Physiol 2010;224:540548.

44 El-Hoss J, Kolind M, Jackson MT, Deo N, Mikulec K, McDonald MM, Little CB, Little DG, Schindeler A: Modulation of endochondral ossification by MEK inhibitors PD0325901 and AZD6244 (Selumetinib). Bone 2014;59:151-161. 


\section{Cellular Physiology Cell Physiol Biochem 2015;36:1753-1766

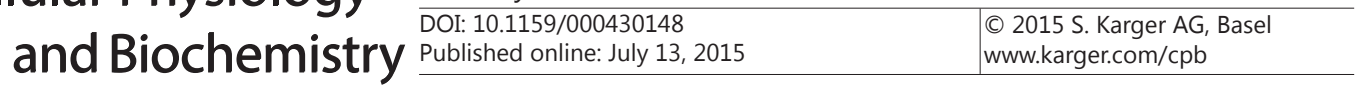

Hou et al.: MiR-381 in Chondrogenesis and Chondrocytes

45 Wang X, Manner PA, Horner A, Shum L, Tuan RS, Nuckolls GH: Regulation of MMP-13 expression by RUNX2 and FGF2 in osteoarthritic cartilage. Osteoarthritis Cartilage 2004;12:963-973.

-46 Chen CG, Thuillier D, Chin EN, Alliston T: Chondrocyte-intrinsic Smad3 represses Runx2-inducible matrix metalloproteinase 13 expression to maintain articular cartilage and prevent osteoarthritis. Arthritis Rheum 2012;64:3278-3289.

47 Qing YF, Zhang QB, Zhou JG, Jiang L: Changes in toll-like receptor (TLR)4-NFkappaB-IL1beta signaling in male gout patients might be involved in the pathogenesis of primary gouty arthritis. Rheumatol Int 2014;34:213-220.

48 Lee AS, Ellman MB, Yan D, Kroin JS, Cole BJ, van Wijnen AJ, Im HJ: A current review of molecular mechanisms regarding osteoarthritis and pain. Gene 2013;527:440-447.

49 Akhtar N, Rasheed Z, Ramamurthy S, Anbazhagan AN, Voss FR, Haqqi TM: MicroRNA-27b regulates the expression of matrix metalloproteinase 13 in human osteoarthritis chondrocytes. Arthritis Rheum 2010;62:1361-1371.

-50 Yamasaki K, Nakasa T, Miyaki S, Ishikawa M, Deie M, Adachi N, Yasunaga Y, Asahara H, Ochi M: Expression of MicroRNA-146a in osteoarthritis cartilage. Arthritis Rheum2009;60:1035-1041.

-51 Tardif G, Hum D, Pelletier JP, Duval N, Martel-Pelletier J: Regulation of the IGFBP-5 and MMP-13 genes by the microRNAs miR-140 and miR-27a in human osteoarthritic chondrocytes. BMC Musculoskelet Disord 2009;10:148.

-52 Jones SW, Watkins G, Le Good N, Roberts S, Murphy CL, Brockbank SM, Needham MR, Read SJ, Newham $\mathrm{P}$ : The identification of differentially expressed microRNA in osteoarthritic tissue that modulate the production of TNF-alpha and MMP13. Osteoarthritis Cartilage 2009;17:464-472.

-53 Vincenti MP, Brinckerhoff CE: Transcriptional regulation of collagenase (MMP-1, MMP-13) genes in arthritis: integration of complex signaling pathways for the recruitment of gene-specific transcription factors. Arthritis Res 2002;4:157-164.

-54 Iliopoulos D, Malizos KN, Oikonomou P, Tsezou A: Integrative microRNA and proteomic approaches identify novel osteoarthritis genes and their collaborative metabolic and inflammatory networks. PLoS One 2008;3:e3740.

-55 Reiter LA, Freeman-Cook KD, Jones CS, Martinelli GJ, Antipas AS, Berliner MA, Datta K, Downs JT, Eskra JD, Forman MD, Greer EM, Guzman R, Hardink JR, Janat F, Keene NF, Laird ER, Liras JL, Lopresti-Morrow LL, Mitchell PG, Pandit J, Robertson D, Sperger D, Vaughn-Bowser ML, Waller DM, Yocum SA: Potent, selective pyrimidinetrione-based inhibitors of MMP-13. Bioorg Med Chem Lett 2006;16:5822-5826.

-56 Latronico MVG and Condorelli G: Therapeutic use of MicroRNAs in Myocardial Disease. Curr Heart Fail Rep 2011;8:193-197.

-57 Kolfschoten IGM, Rogglit E, Nesca V, Regazzi R: Role and Therapeutic Potential of microRNAs in Diabetes. Diabetes Obes Metab 2009;11:S118-129.

58 Yeung ML and Jeang KT: MicroRNAs and Cancer Therapeutics. Pharm Res 2011;28:3043-3049.

59 Kanwal N, John P, Bhatti A: MicroRNA-155 as a therapeutic target for inflammatory diseases. Rheumatol Int 2013;33:557-560. 